\title{
Training laboratory primates - benefits and techniques
}

\author{
K. Westlund \\ Astrid Fagraeus Laboratory, Comparative Medicine, Karolinska Institute, 17177 Stockholm, Sweden \\ Correspondence to: K. Westlund (karolina.westlund@ki.se)
}

Received: 21 September 2015 - Revised: 24 November 2015 - Accepted: 2 December 2015 - Published: 21 December 2015

\begin{abstract}
This review discusses the benefits of training in the effective management of laboratory-housed nonhuman primates, including improved welfare, facilitated husbandry, improved quality of data, and human-animal relationships. Training implies that the animals cooperate in aspects of their own care and is a type of enrichment. Some refined ways of using negative reinforcement are discussed, as well as management perspectives on laboratory primate training. Several approaches to dealing with fear are described: systematic desensitization/counterconditioning (SD/CC) versus combined reinforcement training (NPRT). In addition, a detailed shaping plan covering target training, useful when e.g. moving, weighing, or stationing animals, is presented.
\end{abstract}

\section{Choosing the optimal training approach}

In recent years, laboratory nonhuman primate care has undergone some refinement by the use of systematic training and behaviour management approaches.

Many daily interactions with captive animals involve learning and therefore have a training component: the caretaker pushes a cart containing food into the primate room. She then places food in one compartment and opens the gate to allow the animals access. One animal stares threateningly at his cage-mate, moves into the compartment, and starts feeding. This simple interaction actually involves training, albeit unintentional. The animal's behaviour is impacted through learning: certain events predict other events (the sound of the cart predicts food arrival: classical conditioning); behaviour has an effect on the environment (threats lead to feeding alone: operant conditioning). By acknowledging this fact and using a conscious and deliberate implementation of training, faster and more reliable results can be obtained. In addition, unintended consequences of learning, such as unwittingly rewarding undesired behaviour, may be avoided.

Operant conditioning offers two basic approaches for behavioural management: training using negative reinforcement (NRT), also known as escape/avoidance, and training using positive reinforcement (PRT). Punishment is generally not as effective and is not recommended, for reasons explained below.
Using NRT, animals comply in procedures in order to avoid an aversive stimulus. For example, an animal may move from one part of the enclosure to another in order to evade a person. With PRT, animals have the opportunity to voluntarily cooperate to gain access to a resource, such as shifting to another location in order to obtain treats. There are many reasons to gear the training paradigm towards a positive reinforcement approach rather than using coercion, but sometimes some negative reinforcement may be unavoidable.

In addition, negative punishment (NP) (the removal of the opportunity of earning positive reinforcers as a result of unwanted behaviour: typically a so-called timeout) may be a useful technique in some cases. Following the example above, NP would entail removing treats if the animal does not leave the first part of the enclosure. Positive punishment (PP) involves adding an aversive stimulus as a result of unwanted behaviour. In our example, PP could be that the animal gets sprayed with water if it does not leave the first part of the enclosure. Note that neither type of punishment tells the animal what to do, it simply teaches what not to do. Furthermore, PP is ethically questionable, unnecessary in most cases, has potentially serious side effects, and should therefore be avoided.

Before formal operant training begins, however, it is useful to spend some time establishing a relationship with the animal. 


\subsection{Human-animal relationships (HAR)}

Non-human primates are undomesticated, wild animals (not tame even when bred in captivity) and usually they do not have an inherent affiliation to humans. Early training interaction is about establishing a rapport, habituating the animal to human contact. Developing a positive human-animal relationship opens the door to future training successes. It gains safe access to individuals - even when housed in groups and the animal's potential for voluntary cooperation in medical procedures as well as husbandry situations. Thus, more aversive forms of constraint and coercion can be reduced or eliminated. Positive human interaction serves as a buffer that reduces stress reactions in routine procedures - even before operant training has started. Previous studies have shown that $10 \mathrm{~min}$ a day of positive interactions and treat provisioning will reduce aggressive responses to humans and the occurrence of abnormal behaviour outside the HAR context in group-housed chimpanzees (Baker, 2004), whereas 6 min per week may be insufficient for singly housed macaques (Baker et al., 2010). Another study showed that marmosets exposed to PRT were less stressed by a common procedure (weighing) than control animals (Bassett et al., 2003). Establishing a relationship per se is thus worthwhile even if no further training is attempted. A patient, calm approach is beneficial, keeping the voice low and movements slow and deliberate. Simply hand-feeding animals is thus a good practice to include in regular human socialization programmes (Wolfle, 1985).

When training an animal consistently, a bond develops. The process enhances both the behaviour and the motivation to perform the behaviour, all within the context of a positive interaction. The caretaker gets to know the animal and its preferences, temperament, and physiology. As the observational skills are honed, the trainer may notice small signs indicating health problems long before someone else. The quality of animal care thus benefits from training. In addition, risks to trainers and handlers may be reduced, since trained animals are more predictable and less aggressive (Fig. 1).

Non-human primates communicate with facial expressions and postural displays, in addition to auditory and olfactory cues. Therefore, it is important to come across as nonthreatening or neutral. Loud voices, laughing, staring, and fast movements can all be unsettling or even threatening to a primate. It is important to know the basic behavioural repertoire indicating affective state (in primates ranging from head bobbing to showing the teeth, presenting hind quarters, and scenting with urine) of the species in question (see Wolfensohn and Honess, 2005). However, the caretaker can try to mimic friendly contact sounds or facial expressions (e.g. lip smacking) of the species in question. A word of caution when working with primates: avoid getting caught up in a power struggle and try not to compete for rank with a monkey. The animal should respect the caregiver but not try to fit the person into its own social hierarchy.

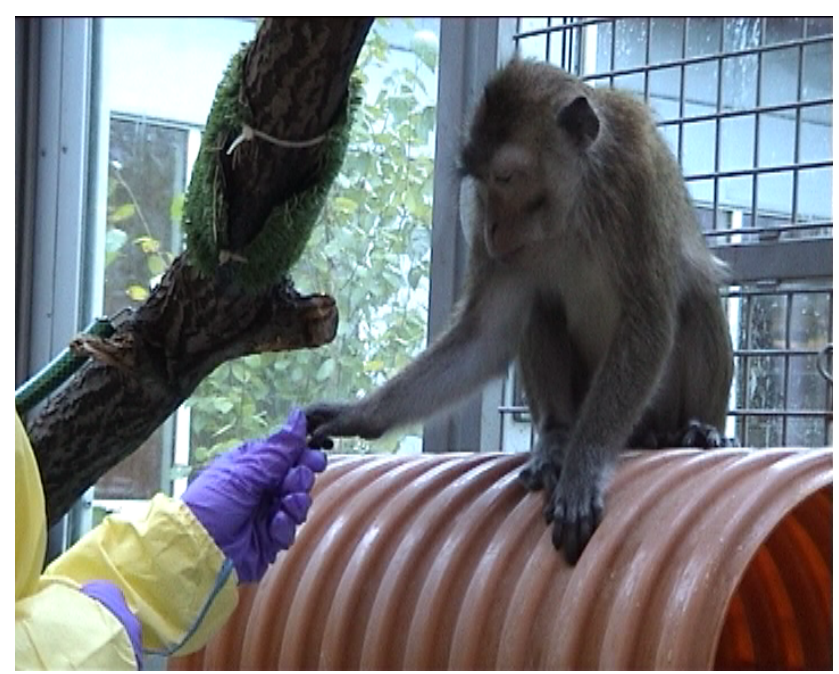

Figure 1. Caretaker interacting with long-tailed macaque (Macaca fascicularis). Image courtesy of Astrid Fagraeus Laboratory.

\subsection{Positive reinforcement training}

In PRT, animals are rewarded for performing desired behaviours rather than punished for doing undesirable behaviours (Fig. 2). The trainer strives to ignore the unwanted while focusing on the correct behaviours. As soon as a desired behaviour is shown, the trainer rewards and thereby reinforces it. Reinforcement actually implies that behaviour is strengthened by rewarding. The next time around, the animal will likely show the behaviour more strongly, faster, and more energetically. Successful PRT is thus a joint effort between trainer and animal, and if behaviour does not change, reinforcement has not occurred.

Through PRT, the animal is provided with choice, control, and a chance to work for food - factors associated with enhanced psychological well-being. In a laboratory setting, this is of particular importance, since stress triggers physiological reactions that might impact research (see below).

Positive reinforcement training does not require food or water deprivation to achieve results. In some disciplines, such as in the field of behavioural neuroscience, the norm has been to use deprivation to entice animals to participate in sessions involving hundreds or even thousands of repetitions. This approach has been under some debate, and alternative approaches to increasing the value of the reward, such as using conditioned reinforcers, variable ratio schedules, reinforcer variability, and reinforcer control, have been suggested (Westlund, 2012a, b).

When animals start learning through PRT, at first progress may be slow. However, as the animal understands the contingency of training (reinforcement conditional on responding to specific cues with the desired behaviour), the "moment of illumination" strikes and the animal can quickly assimilate more behaviours and cues. Often, simple behaviours can 

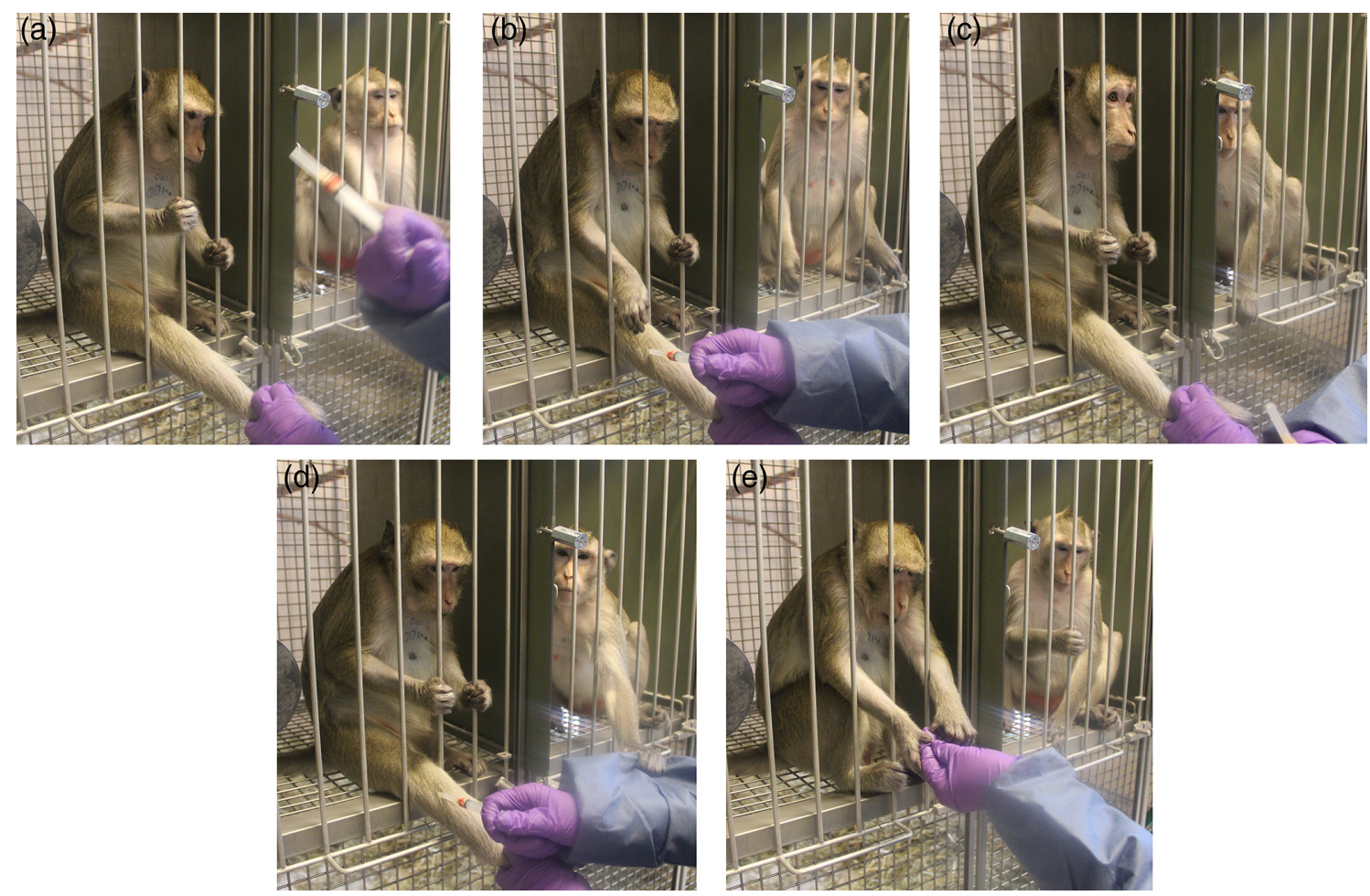

Figure 2. PRT training sequence in a long-tailed macaque (Macaca fascicularis). At this stage in training, the animal is already holding the bars, presenting the right leg and allowing the trainer to grasp it. (a) The trainer presents a capped syringe: the animal is holding on to the bars. (b) The animal lets go of the bars and reaches for the syringe, an unwanted behaviour. (c) The trainer withdraws the syringe; the animal recovers the desired position (holding the bars). (d) The trainer presents the syringe again; this time the animal holds on to the bars and accepts being touched by it. (e) The animal receives positive reinforcement: treats. Note that bar holding is a type of targeting (see below) and also a behaviour incompatible with reaching for the needle - it is impossible to do both at the same time. Unwanted behaviour is not simply ignored: removing the syringe (c) negatively punishes the unwanted behaviour (the chance of earning reinforcement is postponed). Also, at this stage of training there is no needle on the syringe. The neighbour is also keen on training but presently ignored. Image courtesy of Astrid Fagraeus Laboratory.

be used to help the animal move quickly through the confusion - once they understand the training concept, more advanced behaviours can be taught in the later stage of accelerated learning. Below, a simple behaviour shaping plan is demonstrated (target training).

\subsubsection{Using a conditioned reinforcer}

Feed the animal in the transport box, and it will start voluntarily entering the box in anticipation. With primary reinforcers (such as treats) general learning occurs: "I'll stay around and good things will come my way".

You might ask yourself what the point is in adding a conditioned reinforcer such as a clicker, whistle, or a verbal "good" that bridges the interval between the behaviour and the delivery of the primary reinforcer. What is wrong with just food or praise? The animal enters the box and hears a click followed by the delivery of a treat. Could we not just skip the click? Interestingly, animals learning with PRT and a conditioned reinforcer learn faster and remember better than animals learning with only primary reinforcers due to activation of the core emotional SEEKING system (e.g. Pryor, 2009). They also seem to enjoy the training process immensely. Conditioned positive reinforcers (but not primary reinforcers to the same degree) also trigger a dopamine cascade which affects the brain as an "anticipation of good news". This "training elation" indicates a positive affective state and has been systematically studied in dogs, where it includes yipping, tail wagging, jumping and knocking things over, and in rats, where it includes heightened respiration, increased activity, head turning, and increased hormonal output, indicating that the SEEKING mode is activated (Panksepp, 1998). A laboratory monkey trained using PRT and a clicker will eagerly await cues from the trainer, give eye contact (normally unsettling and aversive for primates; de Waal, 1989) and perform learned behaviours to receive a click and a tidbit (Laule, 2010; Prescott et al., 2005).

Importantly, correction involving strong aversives (positive punishment and negative reinforcement) switches the brain from SEEKING mode to avoidance and fear (Dickinson and Pearce, 1977; Pryor, 2009). Thus, by introducing 
punishment, you risk poisoning the whole process and the SEEKING system shutting down. This will result in slower learning and less good retention. You risk the animal quitting on you while training and being more susceptible to distractions.

Even though animals have learned to perform a behaviour, some behaviours or procedures are more demanding than others. For instance, needle training (teaching the animal to stay still and allow the insertion of a needle for a blood draw or injection) may be considered a work always in progress, as regression is common. Proper counterconditioning techniques paired with systematic desensitization (see Box 1) are vital in achieving fast results and minimizing regression.

To summarize, ideally, keep the animal in SEEKING mode by training using PRT and avoiding frightening the animals. In addition, use a conditioned reinforcer, such as a clicker, to get speed of learning and good retention. The animals should reward you with eager anticipation and collaboration.

\subsubsection{A PRT mindset}

Sometimes it is difficult to reach all animals. They may be housed in big corrals or big groups. There might be restrictions on the types or amounts of food animals are allowed to receive or what type of physical activity is available to them. If the setting does not allow for extended training practices, a mindset that is geared towards a positive human-animal interaction is still invaluable.

Laule (2010) recommended some valuable strategies to this end involving planning ahead, preparing for research protocols and gaining the cooperation of the animal in as many steps of the research protocol as possible. Also, employing the least invasive methods to achieve objectives; an oral medication can be given in a tasty substance that is ingested voluntarily, thus avoiding a potentially aversive gastric gavage.

Incorporate an element of training into daily routines. If the setting does not allow for regular training sessions, learning opportunities can still be taken. If a specific device is to be used during a scientific procedure, bring it into the animal room and reward the animals for calm behaviour in its presence. Do these types of interactions when interactions were occurring anyway, such as during cleaning, feeding, or visual inspections. By identifying individual components of the final behaviour, and working on these relatively small units on a daily basis, the animal is slowly conditioned to accept and cooperate with the process. Even if the final behaviour is not trained in its entirety, there is a greater likelihood that the animal will be more cooperative and less fearful when the actual procedure is implemented. Like humans, animals learn through repetitions. Find ways to train frequently and regularly (adapted from Laule, 2010).

\subsection{Negative reinforcement}

NRT has a bad reputation in animal training and is often avoided as a training tool since traditionally it has involved pain, discomfort, or unpleasant experiences (e.g. Laule, 2010). However, a recent study used the slow and systematic presentations of novel objects at some distance from the animals to trigger avoidance behaviour. Importantly, the removal of the novel objects was contingent on the animal's behaviour and combined with positive reinforcement (see the procedure outlined below, adapted from Wergård et al., 2014) This approach resulted in the successful training, with minimal exposure to aversives, of rhesus macaques to be enclosed in a smaller section of their cage - a feat that could not be achieved with control animals trained with PRT alone during the same time interval. Thus, low-intensity uses of NRT in combination with PRT (in the following abbreviated as NPRT) may be one approach to obtain objectives that are unfeasible with PRT alone.

When NPRT is used e.g. for teaching animals to be enclosed in a small section of the cage, and exclusive PRT is not an option, here are some ways of lessening the impact of the aversive stimuli (adapted from Wergård et al., 2014).

- Build a relationship between trainer and animal, as mentioned above. If aversive stimuli are to be used during training, it is vitally important that the risk of fear conditioning is reduced by establishing trust first.

- Conduct "pure" PRT sessions outside the NPRT sessions. Aversive elements should be the exception, not the norm.

- Introduce aversive elements gradually, using systematic desensitization and counterconditioning (SD/CC) (Box 1).

- Use signals predicting aversive elements, as this reduces stress (e.g. Basset and Buchanan-Smith, 2007). Establishing a command before presenting a negative reinforcer enables the monkey to avoid the aversive altogether by performing the correct behaviour once it has learned what to do. No command implies that the monkey must escape the aversive - it has less control than if it avoids it. If multiple animals are being trained in the same room: use unique signals for each animal or groups of animals.

- Use aversive stimulation of as low intensity as you need. In other words, do not shout and spray water to make an animal move. Rather, show a novel object at some distance; at the very moment the animal leans away from the object, remove it.

- Offering a positive reinforcer when the animal performs a correct response counterconditions the aversive elements of the process, rendering it less aversive. When 
the animal leans away from the novel object, give him a treat in the direction you want him to move.

While NPRT may be useful, the fact that the procedure contains aversive elements means that there is a risk of frightening animals, which may impact welfare negatively. Combined reinforcement is thus not a beginner's tool and should not be the first approach for the type of situation described above.

\section{Using careful NPRT to reduce fear}

This intricate use of combined reinforcement (NPRT) serves to acclimatize frightened animals to human contact. Frightened animals want to increase the distance to the scary stimulus: the trainer. When they are in a cage, they cannot move very far - but the trainer can reinforce desired behaviour by moving away.

- Place an assortment of tasty treats on surfaces near the cage front and back away far enough so that animals are not freezing from fear. Initially, the animals will likely jump away when approached. Avoid direct eye contact.

- As animals make any movement, back away even further until they have eaten the treats.

- Refill the treats and repeat the procedure, backing away contingently on animals' movement.

- Gradually switch so that backing away is contingent specifically on approach, not just any movement. Soon the animals may approach deliberately to make the trainer go away so they can get to the treats.

- As the animals gain confidence, gradually shape the distances shorter so that the initial as well as the final position are progressively closer to the cage.

- When the trainer is no longer backing away, offer the treats and let one hand remain hovering nearby the treats until the animals move forward - then slowly remove the hand. Repeat.

- Try offering the food out of the hand.

This procedure may be successful quickly if the animals are interested in the food and moving. If carried out well, it is not particularly aversive to the animals since they are in control - by moving towards the trainer, they make the scary person go away, and get fabulous treats too!

\section{The benefits of having a "handling and training plan"}

\subsection{Improved welfare and facilitated husbandry}

Training is an important refinement tool and has been used to improve husbandry procedures such as moving and weighing animals (McKinley et al., 2003). Also, introductions and

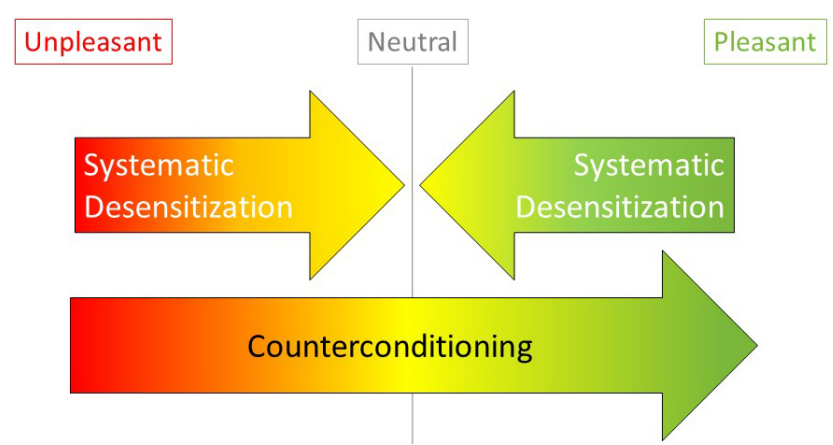

Box 1. Addressing fear: systematic desensitization and counterconditioning (SD/CC).

Through counterconditioning combined with systematic desensitization, stress can be reduced, including physiological as well as behavioural responses. Briefly, counterconditioning usually involves pairing an aversive stimulus with a treat, thus reducing the unpleasantness of the stressor (e.g. Clay et al., 2009). Used alone, systematic desensitization reduces stimuli to neutral, whereas counterconditioning converts adverse stimuli to something that the animal looks forward to.

Systematic desensitization does not entail associative learning but is simply exposure to initial low-intensity versions of the stimulus, all the while staying below the threshold where the animal shows an escape reaction. The intensity of the exposure is gradually increased until the full exposure, without fear reaction, is reached. An example may be to systematically desensitize the animal to being touched by a needle. Initially, the animal may be very frightened of the needle and show an escape reaction from merely seeing it in the hand of the trainer. The first steps in the desensitization process may then be to carry the syringe in a pocket, barely visible. If the animal shows no reaction, the syringe is gradually introduced over the course of several training sessions and brought closer and closer to the animal. Finally, the animal will accept being touched by the syringe without the needle. Then the needle is attached and the process repeated (quicker this time, usually)

This technique is ideally combined with counterconditioning: when the aversive stimulus is perceived by the animal, a treat is given. Through this procedure, the aversive stimulus becomes a conditioned positive reinforcer: when the animal sees the needle, it will expect a treat.

socialization with conspecifics may be facilitated (Fritz and Howell, 2001). Veterinary care benefits from an increased access to conscious animals standing still or presenting limbs for inspection, and training enhances the voluntary cooperation in the collection of blood, semen, and urine (Coleman et al., 2008; Keller, 1988; McKinley et al., 2003). It has been used to reduce stereotypical behaviour and other abnormal behaviour such as self-injurious behaviour, reduce aggression, enhance enrichment programs, and increase personnel safety (e.g. Prescott et al., 2005; Fig. 3). 


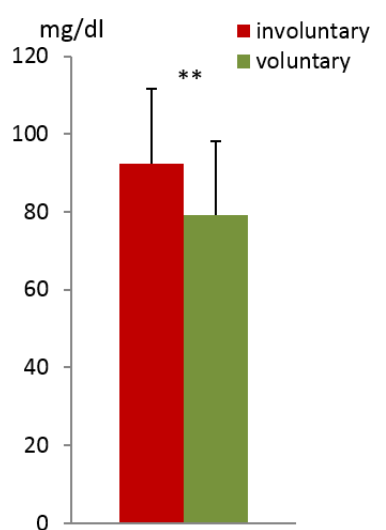

(a) - blood glucose

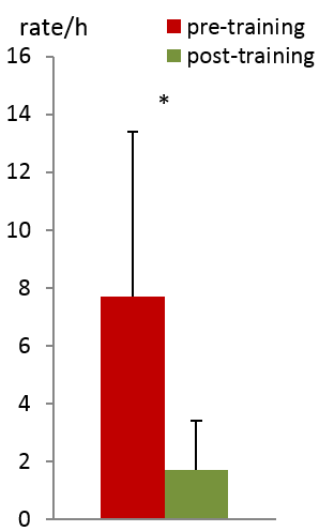

(b) - aggression at feeding

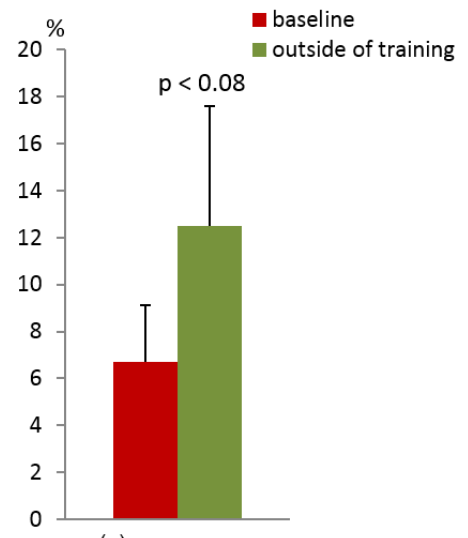

(c) - affiliation

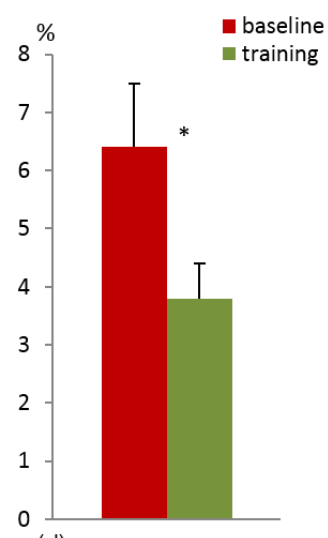

(d) - abnormal behaviour
Figure 3. (a) Mean (+ SD) blood glucose (GLU) levels $\left(\mathrm{mg} \mathrm{dL}^{-1}\right.$ ) in 79 chimpanzees presenting a thigh either voluntarily or involuntarily for an anaesthetic injection (dependent data; Lambeth et al., 2006). (b) Mean hourly rate of aggression (+ SE) before and after training during feeding in a group of eight chimpanzees (Bloomsmith et al., 1994). (c) Mean percent time (+ SD) spent in affiliative interactions for seven low-affiliating rhesus macaques during baseline and outside of training during the training phase (Schapiro et al., 2001). (d) Mean percent time (+SE) spent in abnormal behaviour in a group of 12 chimpanzees before and during implementation of a training program (Pomerantz and Terkel, 2009). Reprinted with permission $\left(^{*}=p<0.05\right.$; $\left.^{* *}=p<0.01\right)$.

Trained animals show a remarkable reliability in participating in known procedures. Once learned, behaviours are usually maintained (Fernström et al., 2009, found that after a break of 27 days of no training, only 2/33 female rhesus macaques had regressed - 7/33 actually performed better after the break). Many procedures involve restraint in untrained animals, which is potentially stressful. However, animals are less stressed when allowed to voluntarily cooperate, as evidenced by a reduction in cortisol levels, stress-related abortions, physical resistance to handling, and fear responses such as fear-grinning, screaming, and acute diarrhoea (re- viewed in e.g. Laule et al., 2003) - training thus increases welfare.

\subsection{Improved quality of data}

Apart from ethical or practical reasons for attempting to train laboratory primates, there are strong reasons from a scientific perspective to endeavour to train animals. The most commonly used procedure when teaching the animal to deal with new situations in the environment is habituation (simply exposing an animal to a stimulus until it stops responding to it). However, after habituation animals may seem behaviourally unaffected by aversive stimuli but still react physiologically. After repeated chairing of monkeys, behavioural changes occur - the animals stop responding with escape attempts and vocalizations. The animals may have habituated behaviourally, but data show that the physiological stress response is maintained, albeit somewhat diminished (Ruys et al., 2004). In other words, docility from the animal can occur with the animal still being stressed, and learned helplessness may be confused with compliance (Mineka, 1982; see Box 1 and Sect. 1.3 (p. 5) on NPRT for ways of addressing this issue.)

As stress engenders a physiological reaction leading to systemic effects e.g involving. respiratory, reproductive and gastrointestinal function, neurology, immune function, and learning (Conrad, 2010; Klonoff et al., 1976), scientific studies risk being influenced by effects due to stress rather than the procedure itself. Stress may impact the quality of research in two ways (Boscarino, 1997; Lazarus et al., 1985; Strekalova et al., 2005). Animals react to stressors differently, and an increase of variability in results and reduced statistical power may be one effect of stress on the experiment, leading to a difficulty in seeing effects of treatment and the need for an increased number of animals to reach statistical significance. In addition, stress may be a confounding factor in certain types of research (e.g. Capitanio and Lerche, 1998). This impact will vary widely depending on what kind of data are collected (Lazarus et al., 1985). All types of studies involving areas where stressors have an impact could potentially be affected. If stress can be prevented or reduced through training, better quality data may be the outcome. Additionally, stress reduction is a type of refinement and thus of importance from a $3 \mathrm{R}$ perspective.

Ideally, in an experimental situation, the only factor affecting an animal should be the experimental variables and nothing else. Proper acclimatization (adaptation to environmental conditions), including training, is therefore of great importance. Since training reduces stress and therefore diminishes variability, it can proceed even when the experiment has started when control groups are used. Any differences between controls and experimental groups could then be ascribed to experimental treatments. If, however, the animal serves as its own control, one should keep the potential physiological effects of training in mind when examin- 
ing data if training continues throughout the experiment. Any differences between control data and experimental data could then be attributed to experimental treatments - or potentially to training effects.

\subsection{Training as enrichment}

A good enrichment intervention should do the following (Westlund, 2014):

- give the animal control over its environment,

- add behavioural choices,

- promote species-specific behaviour, and

- empower the animal to cope with challenges.

Allowing the animal training opportunities can be considered as providing a type of enrichment since all four of these objectives are achieved. Also, there may be additional effects beneficial to welfare that cannot be obtained by traditional enrichment alone. For instance, reducing fear through systematic desensitization and counterconditioning (see Box 1) as well as improving human-animal relationships (above), facilitating husbandry procedures, and using functional assessments to address problematic behaviours through training (Hastings and Brown, 2000).

\subsection{Animals participating in their own care}

By the very nature of operant conditioning (learning from the consequences of a behaviour), the animal learns to affect its experiences. Once cues are learned, the animal can choose to respond to them or not to comply. This choice per se empowers the animal, as a certain measure of control is achieved - indeed control can be construed as a primary reinforcer something the animal is willing to work for (Finkelstein and Ramey, 1977)! It is up to the trainer to make sure that complying with a request is worth the animal's while. Ideally, the animal should always be given a choice. It might still be exposed to the procedure should it choose not to comply this time, but it might learn something and comply the next time around. There is ample evidence that being able to control aspects of the environment reduces stress in captive animals (Moberg, 2000).

In training, one thing to keep in mind is what the animal's preferences are when choosing a training position. Arboreal species enjoy observing their surroundings from an elevated platform, looking down. When attempting to train an animal, it is often wise to start when the animal is in a position with a sense of control, such as perching in a favourite elevated platform, or in companionship with favourite cage mates although training several animals in a group may entail challenges. Isolation of social animals is a potential stressor and must be used conscientiously.

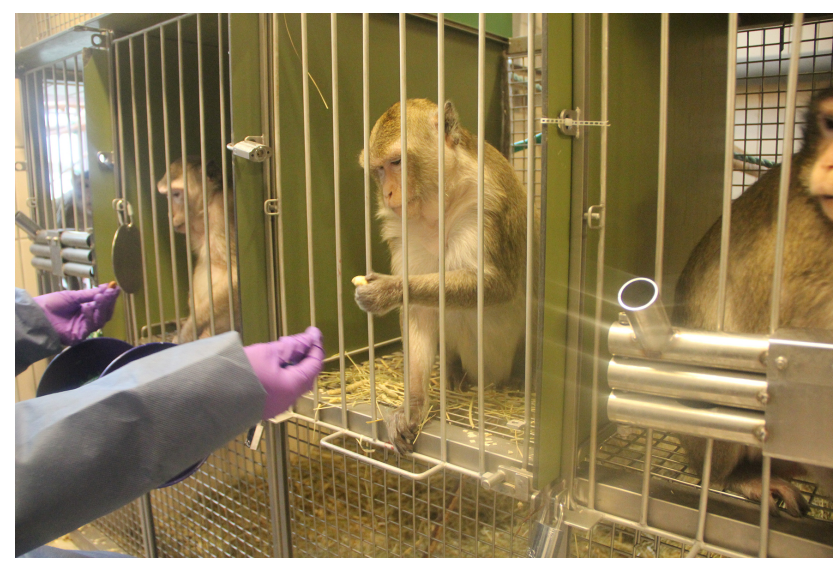

Figure 4. Visual barriers may separate a group of animals during training sessions. Here, the animals are in a training tunnel adjacent to their indoor/outdoor cages and may leave their training positions at any time. Image courtesy of Astrid Fagraeus Laboratory.

\section{Management perspectives}

\subsection{Factors affecting training efficacy}

In cases where extended training practices are feasible, it is useful to consider some factors affecting the efficacy of training.

Firstly, the animals' temperament can be crucial. Studies have shown that a simple novel-object test can reveal temperamental differences between animals that influence initial trainability (Coleman et al., 2005): animals who investigated novel objects were more easily target trained than inhibited animals. It is our observation that bold animals who dare take treats from a trainer's hand also learn initial training tasks quicker, probably since they do not have to spend much time overcoming fear of the trainer (Wergård, 2015).

Secondly, group size will most probably influence trainability. Since there is a social dominance hierarchy in most captive primate groups, there will be a differential access to resources among animals. Since the trainer will become an attractive resource, it is not unusual that dominant animals attempt to monopolize the trainer in the initial stages of training, and it is often easiest to start with the dominant individual. Being able to temporarily separate animals (Fig. 4) or use multiple trainers might be two ways to overcome this potential problem. Also, dominant animals can learn to allow subordinates feeding and training opportunities. With the use of positive reinforcement (for allowing the subordinate to feed), extinction and the occasional timeout in response to agonistic behaviour, the dominant animal becomes less aggressive and more tolerant. Essentially, he learns that stationing pays off and chasing cage-mates does not. Meanwhile, the subordinate animal becomes less fearful and more willing to accept food items in the presence of the dominant animal. This type 
of training is often referred to as cooperative feeding (e.g. Laule, 2010).

Cooperative feeding can be used to facilitate introductions: during an episode of non-contact familiarization, the dominant animal can learn that the reception of food is directly related to whether he allows the subordinate to feed.

Husbandry training sessions may range from 1 to perhaps 20 min depending on the engagement of the animal, progress made, and satiation. Sessions benefit from being separated by a sleeping opportunity: it is counterproductive to invest in more than one training session (teaching the same behaviour) a day - the latter training session may interfere with memory consolidation of the first (see discussion in Fernström et al., 2009). During sleep, memory is consolidated and learning occurs.

Captive monkey groups are sometimes required to move in single file from one compartment to another in order to be captured. Observation has shown that animals often maintain the same position within the file with remarkable consistency. This progression order varies depending on whether the behaviour is prompted or natural. The prompted progression (involving coaxing and negative reinforcement) may be a function of age (older animals moving first, having learned what is expected of them and overcoming the fear involved), whereas the natural progression order in another study was equally stable but unrelated to sex, age, or rank in rhesus macaques (Reinhardt and Reinhardt, 1987; Reinhardt, 1992). The implication here is that animals often develop a predictable order in which they move from one compartment to another, which has managerial value since it allows one person to quickly access a targeted animal. Pairing such a progression order with positive reinforcement in a tunnel adjoining two areas has been used as a means of accessing all individuals even in large groups (Veeder et al., 2009).

\subsection{Safety, skill and setup}

In order for training to be efficient, several factors are important. Monkeys are typically xenophobic and may not be comfortable with strangers handling them. Veterinarians and scientists should therefore be introduced to animals in a training context to avoid fear conditioning, which may impact research. Additionally, it is beneficial if cages allow for easy training access (Fig. 5).

In some facilities, open contact (without a barrier between trainer and animal) is used, whereas in others, protected contact (a barrier between trainer and animal in place) is prevalent. The risk assessment involves known and unknown diseases, animal history and behavioural profile as well as caretaker experience and proficiency in reading the animal and reacting appropriately. Calm and confident behaviour is important regardless.

Additionally, the personnel involved in training need the required skill and experience to apply reinforcement techniques and systematic desensitization and countercondition-

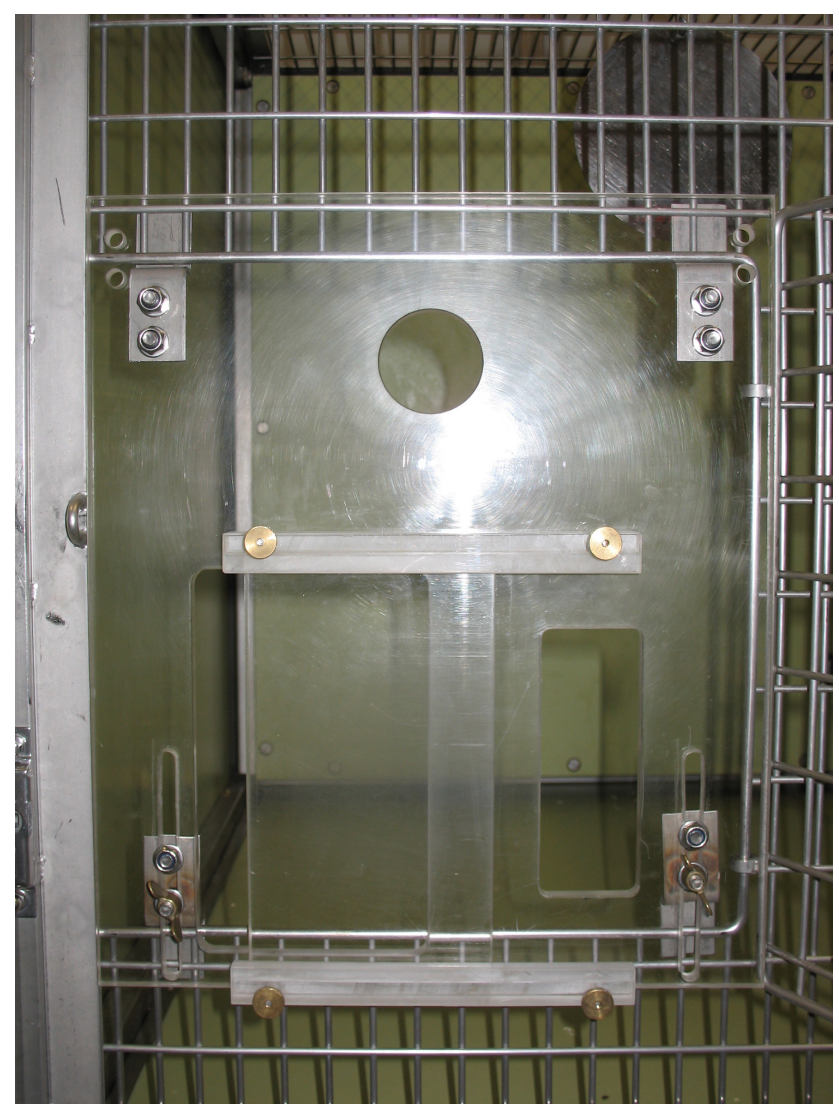

Figure 5. Cages may have to be changed to accommodate training practices - here a plexiglass "door" allows protected contact with the animal. Image courtesy of the German Primate Center (DPZ).

ing. Commitment and time are other important factors: the animals learn through repetitions, and if training occurs too seldom, learning is slowed down. When learning slows, the motivation of the trainer is affected, and training may occur even more infrequently. Conversely, successful training inspires one to find time for more training sessions, and both negative and positive training spirals are common. Having a set time for training (e.g. early afternoons on certain days) may be an approach to firmly incorporate training into weekly routines. Sharing training successes and receiving encouragement from management are other important factors.

In facilities where training is done systematically, the most commonly used approach is to have one or several skilled trainers doing the most advanced training (including someone overseeing and organizing training activities, and problem solving). If the main caretaker is knowledgeable about training, allowing that person to do the training is probably best because of the benefits of HAR. All caretakers benefit from knowing some basic training techniques and may sometimes ask animals to present a behaviour that someone else trained. Usually, it is best if one person trains a behaviour before "transferring" it to someone else - behaviour generalizes reasonably well to other people provided trainers use 
the same cues and have no aversive history. Agreement and consistency among trainers will promote the success of the program.

\section{Conclusions}

To summarize, training is an important aspect of good animal behaviour management as it reduces stress, promoting both animal welfare and the quality of science. Key points for a successful program include, but are not limited to, engaged trainers, open-minded researchers, and supportive upper management. Additionally, learning from outside the guild of laboratory primate trainers is very beneficial, e.g. a cross-fertilization from zoo and pet trainers, behaviour analysts, ethologists, and researchers from the field of affective neuroscience. 


\section{Appendix A: Basic behaviour - target training}

Aim: training an animal to touch a target, such as a shoehorn or part of the cage, on cue. Application: target training helps the animal learn the training contingency. It is used in cooperation training for stationing and moving animals. This is a useful first behaviour to be trained because of its applicability and also because the animal moves!

Work with animals individually and together. At first it might be easiest not to separate animals when training. Choose a location where the animals feel safe, a preferred spot. The set-up might include the trainer sitting down.

Focus on the individual that dares approach. This is simplest in those groups where the most daring animal is also the dominant. If the most daring animal is afraid of another group mate, try to separate them or make sure there are visual barriers. Then start training the most daring animal. If the animals get agitated when you separate them, habituate them to this procedure first (that is, separate them every day for a week and let them out again). In a group setting, it is suggested that the cue involves the animal's name.

\section{Shaping plan:}

- Click once and give the animal a treat. Repeat 5-10 times. Note: click before even showing the treat; the sound should predict the treat. The two should not be presented simultaneously.

- If the animal seems afraid of the clicking sound, try lowering the sound level, e.g. by pocketing the clicker.

Level 1: the animal is probably classically conditioned and has learned that click means treat.

- Choose a target - preferably one that you do not attach, such as a shoehorn. Take notes on the specifics of the target for that particular individual. Each individual should have an individual target. (Consider whether the animal can see colour; e.g. marmosets are colour blind!)

- Click and treat if the animal looks at the target. Click and treat if the animal moves toward the target.

- Click and treat if the animal touches the target - regardless of body part initially. Repeat only a few times.

- Once the animal is more interested in the target, wait until he touches it with either hand before clicking and treating. If he does not seem to get interested, repeat Level $1 \mathrm{a}$ few more times. Consider: is his hearing impaired?

- If the animal does not show any behaviour that can be reinforced, try a distraction. Put a piece of sawdust on the target, as this might induce the animal to look at it or even touch it. If it does not work, try a lure. If needed, do a preference test (offering a number of different treats - switching locations in each presentation). Then put a desirable treat on the target and click when the animal takes it. After 2-3 times, present the target without the lure and try to click if she looks at it or moves toward it as described above. Note the risk of using lures: they should not be a consequence of not cooperating; in that case she learns not to cooperate $-2-3$ times maximum!

- If the animal is afraid of the target, stand as far away as you can without him showing any signs of fear when you display the target. Display the target, click and remove it, and give treat. Gradually step closer (systematic desensitization and counterconditioning). Shape the distance until the animal is comfortable with having you display the target next to him. Another option is that you attach the target to his cage for a day or so, so she can examine it at his leisure.

- If the animal gets overexcited and pulls at the target, try a few early clicks (clicking immediately before she touches the target; that slows the motion down).

- At all times, after clicking, remove the target, so that presenting it again is a new trial - and a new cue. Also, feed after each click.

Level 2: the animal touches the target, is operantly conditioned and lets go of the target when you click.

- Consider what you want the behaviour to look like. Left hand, right hand, both, nose? Through differential reinforcement you will make him understand what you want (ignore wrong body parts, reinforce right ones).

- If the animal does not touch the target in $10 \mathrm{~s}$ after presentation, remove it. Wait until she seems focused on you, and then offer it again. Shorten latency until a maximum of $5 \mathrm{~s}$.

- When the animal is consistently touching the target within $5 \mathrm{~s}$, but before it is perfect ( $80 \%$ is enough), add the cue, e.g. "(animal name) - target". The compound cue is both the name and the word and the presentation of the target - say the name first and then present the target as you say "target".

Level 3: you say "(animal name) - target" as you present target, and the animal touches it within $5 \mathrm{~s}$.

- Start presenting the target in different positions in the cage - she should now be moving about to touch the target. Up, down, left, right. Increase distance. Do not forget the cue!

- Make him go to locations that are not preferred, such as on the floor or in a crate. Click and reinforce, repeat. 
Mix this up with "easier" places that are less challenging. If the animal is hesitant to come down to the floor, consider his perception of you - you might be frightening. Sit down, squat, look away.

- Keep a high rate of continuous reinforcement. Click, treat.

- "Feed for position" - one way of getting the animal to scary locations is by feeding there prior to presenting the target in that location.

Level 4: the animal has generalized the behaviour, follows to designated areas in the cage, and touches the target as soon as she can reach it, on at least $80 \%$ of occasions.

- Show another trainer which target is used for which animal, what the behaviour looks like, and discuss if there have been problems. The animal performs the behaviour for the other trainer, who uses the techniques you describe.

Level 5: behaviour is transferred to another trainer.

- For stationary targets that have passed Level 2-4, work on cue control. By now the animal should have heard the word "target" so many times that he associates it to the actual behaviour. It is time to start making sure he only touches it on cue and ignores it if there is no cue (it might be difficult to find the time to say the animal's name and "target" during the learning phase when he grabs the target constantly; in this case, choose a shorter cue).

- She must stop touching the target unless you specifically cued him. She should sit and wait for the cue. Stop clicking if he touches the target unless you have said "target".

- If he anticipates by eagerly going for the target without a cue, wait until after he lets go before immediately saying "target" and clicking as he touches it again. Gradually shape the time you wait before giving cue to $30 \mathrm{~s}$. Add the animal's name so that the cue becomes "(animal name) - target".

- As the rate of reinforcement drops he might become grumpy. Feed away from the target, so there is time to say "target" before he touches it again.

Level 6: stimulus control. The animal does not touch the target until you give cue, and he does so immediately. She does not give any other behaviour on the cue "(animal name) - target", and he does not touch the target in response to some other cue.

- Train the behaviour to fluency by exposing him to distractions: the presence of another person (a), room cleaning in progress (b), and the presence of a scary person (c).
- Despite these distractions, the animal should be completely focused on training. The criterion for success is that he should touch the target within $5 \mathrm{~s}$ in $95 \%$ of cues despite distractions as described above.

Level $7(a b c)$ : Fluency: the animal touches the target within $5 \mathrm{~s}$ in $95 \%$ of cues despite distractions.

Note that during this target training, duration of the behaviour is not trained in order to facilitate stimulus control. Click immediately, always, when the animal touches the target. However, facilitate later duration training by choosing a particular way of presenting the target, such as perpendicular to the cage (and another when duration is required, e.g. parallel to the cage). It might be difficult to generalize the behaviour in subordinates; choose locations that minimize dominance interactions while still testing that the animal has understood the concept of following the target even to places where the trainer might be intimidating. When the animal has achieved Level 4 is the time to transfer the behaviour to other staff, who trains this when the principal trainer is absent. You may also use a stationary target, such as a carabiner. When removing a stationary target, make sure that the animal cannot attack you and cause injury because of frustration (or train a release cue - early in training).

Remember that during shaping it is important that the animal is always doing easy things - do not increase criteria too quickly. Maintain a high level of clicks and reinforcements; if the clicks come too seldom he loses interest. Gradually increase your demands. At first you might try to present the target when the animal is approaching to get the treat. Some animals are willing to "eat and work" at the same time, while others are not. If the animal gets stuck, try lowering the criterion and making it easy for the animal to succeed. Note progress in training record. Levels 4 and 5 should be noted in chart visible to other trainers/caretakers.

\section{Risk assessment:}

- Certain individuals may grab when you feed them - risk of scratching.

Always wear double gloves when working with unknown and infected animals and with animals that you know are grabby.

- When you remove a stationary target the animal may become frustrated - and aggressive.

If the animal shows signs of frustration, close compartment so that he cannot reach your hands when you remove target. Or use a shoehorn to move him away from the stationary target as you remove it. Alternatively - 
train a release cue (early in training!). Rather be safe than sorry - do not take chances!

- The animal might become frustrated when learning stimulus control - suddenly treat rate drops.

If the animal receives too few clicks and become frustrated, shape the waiting interval more slowly. Also, make it easier for him to succeed by using a moveable target rather than a stationary one.

- In general, teach the animal that aggression does not pay off. Give him an LRS (short break) or a timeout (negative punishment).

\section{Prerequisites for target training:}

- Trainer prerequisite: basic skills.

- Animal prerequisite: preferably non-aggressive, relaxed in the presence of humans, and accepting treats from the hand. 
Acknowledgements. Eckhard Heymann, Eva-Marie Wergård, and an anonymous reviewer provided valuable feedback on previous versions of this paper. I also appreciate the financial support from the EUprim-net II research initiative support from the 7th framework program of the EU, grant agreement no. 262443.

Edited by: A. Vitale

Reviewed by: two anonymous referees

\section{References}

Baker, K. C.: Benefits of positive human interaction for sociallyhoused chimpanzees, Anim. Welfare, 13, p. 239, 2004.

Baker, K. C., Bloomsmith, M. A., Neu, K., Griffis, C., and Maloney, M.: Positive reinforcement training as enrichment for singly housed rhesus macaques (Macaca mulatta), Anim. Welfare, 19, p. 307, 2010.

Bassett, L., Buchanan-Smith, H. M., McKinley, J., and Smith, T. E.: Effects of training on stress-related behavior of the common marmoset (Callithrix jacchus) in relation to coping with routine husbandry procedures, J. Appl. Anim. Welf. Sci., 6, 221-233, 2003.

Bassett, L. and Buchanan-Smith, H. M.: Effects of predictability on the welfare of captive animals, Appl. Anim. Behav. Sci., 102, 223-245, 2007.

Bloomsmith, M. A., Laule, G. E., Alford, P. L., and Thurston, R. H.: Using training to moderate chimpanzee aggression during feeding, Zoo Biology, 13, 557-566, 1994.

Boscarino, J. A.: Diseases among men 20 years after exposure to severe stress: implications for clinical research and medical care, Psychosom. Med., 59, 605-614, 1997.

Capitanio, J. P. and Lerche, N.: Social separation, housing relocation, and survival in simian AIDS: a retrospective analysis, Psychosom. Med., 60, 235-244, 1998.

Clay, A. W., Bloomsmith, M. A., Marr, M. J., and Maple, T. L.: Habituation and desensitization as methods for reducing fearful behavior in singly housed rhesus macaques, Am. J. Primatol., 71, 30-39, 2009.

Coleman, K., Tully, L. A., and McMillan, J. L.: Temperament correlates with training success in adult rhesus macaques, Am. J. Primatol., 65, 63-71, 2005.

Coleman, K., Pranger, L., Maier, A., Lambeth, S. P., Perlman, J. E., Thiele, E., and Schapiro, S. J.: Training rhesus macaques for venipuncture using positive reinforcement techniques: a comparison with chimpanzees, J. Am. Assoc. Lab. Anim., 47, p. 37, 2008.

Conrad, C. D.: A critical review of chronic stress effects on spatial learning and memory, Prog. Neuro.-Psychoph., 34, 742-755, 2010.

Dickinson, A. and Pearce, J. M.: Inhibitory interactions between appetitive and aversive stimuli, Psychol. Bull., 84, p. 690, 1977.

de Waal, F.: Peacemaking among primates, Harvard University Press, London, England, 1990.

Fernström, A. L., Fredlund, H., Spångberg, M., and Westlund, K.: Positive reinforcement training in rhesus macaques-training progress as a result of training frequency, Am. J. Primatol., 71, 373-379, 2009.

Finkelstein, N. W. and Ramey, C. T.: Learning to control the environment in infancy, Child Dev., 48, 806-819, 1977.
Fritz, J. and Howell, S.: Captive chimpanzee social group formation, The care and management of captive chimpanzees, 2, 173204, 2001.

Hastings, R. P. and Brown, T.: Functional assessment and challenging behaviors: Some future directions, Res. Pract. Pers. Sev. D, 25, 229-240, 2000.

Keller Jr., K. V.: Training Atlantic bottlenose dolphins (Tursiops truncatus) for artificial insemination, Naval Ocean Systems Center, San Diego CA, 1988.

Klonoff, H., McDougall, G., Clark, C., Kramer, P., and Horgan, J.: The neuropsychological, psychiatric, and physical effects of prolonged and severe stress: 30 years later, J. Nerv. Ment. Dis., 163, 246-252, 1976.

Lazarus, R. S., DeLongis, A., Folkman, S., and Gruen, R.: Stress and adaptational outcomes: The problem of confounded measures, Am. Psychol., 40, 770-785, 1985.

Laule, G.: Positive reinforcement training for laboratory animals, in: the UFAW handbook on the care and management of laboratory and other research animals, edited by: Hubrecht, R. and Kirkwood, J., 8th Edn., Wiley-Blackwell, Oxford, 2010.

Laule, G. E., Bloomsmith, M. A., and Schapiro, S. J.: The use of positive reinforcement training techniques to enhance the care, management, and welfare of primates in the laboratory, J. Appl. Anim. Welf. Sci., 6, 163-173, 2003.

Lambeth, S. P., Hau, J., Perlman, J. E., Martino, M., and Schapiro, S. J.: Positive reinforcement training affects hematologic and serum chemistry values in captive chimpanzees (Pan troglodytes), Am. J. Primatol., 68, 245-256, 2006.

McKinley, J., Buchanan-Smith, H. M., Bassett, L., and Morris, K.: Training common marmosets (Callithrix jacchus) to cooperate during routine laboratory procedures: Ease of training and time investment, J. Appl. Anim. Welf. Sci., 6, 209-220, 2003.

Mineka, S.: Depression and helplessness in primates, in: Child nurturance, Springer US, New York, 197-242, 1982.

Moberg, G. P.: Biological response to stress: implications for animal welfare, in: The biology of animal stress: basic principles and implications for animal welfare, edited by: Moberg, G. P. and Mench, J. A., CABI International, Wallingford, UK, New York, USA, 1-21, 2000.

Panksepp, J.: Affective neuroscience: The foundations of human and animal emotions, Oxford University Press, 1998.

Pomerantz, O. and Terkel, J.: Effects of positive reinforcement training techniques on the psychological welfare of zoo-housed chimpanzees (Pan troglodytes), Am. J. Primatol., 71, 687-695, 2009.

Prescott, M. J., Bowell, V. A., Buchanan-Smith, H. M.: Training of laboratory-housed non-human primates - Part 2: resources for developing and implementing training programmes, Anim. Technol. Welf., 133-148, 2005.

Pryor, K.: Reaching the animal mind: clicker training and what it teaches us about all animals, Simon and Schuster, New York, 257 p., 2009.

Reinhardt, V.: Voluntary progression order in captive rhesus macaques, Zoo. Biology, 11, 61-66, ISO 690, 1992.

Reinhardt, V., Reinhardt, A., and Houser, D.: Prompted progression order in a troop of captive rhesus monkeys, Folia Primatologica, 48, 121-124, ISO 690, 1987. 
Ruys, J. D., Mendoza, S. P., Capitanio, J. P., and Mason, W. A.: Behavioral and physiological adaptation to repeated chair restraint in rhesus macaques, Physiol. Behav., 82, 205-213, 2004.

Schapiro, S. J., Perlman, J. E., and Boudreau, B. A.: Manipulating the affiliative interactions of group-housed rhesus macaques using positive reinforcement training techniques, Am. J. Primatol., 55, 137-149, 2001.

Strekalova, T., Spanagel, R., Dolgov, O., and Bartsch, D.: Stressinduced hyperlocomotion as a confounding factor in anxiety and depression models in mice, Behav. Pharmacol., 16, 171-180, 2005.

Veeder, C. L., Bloomsmith, M. A., McMillan, J. L., Perlman, J. E., and Martin, A. L.: Positive reinforcement training to enhance the voluntary movement of group-housed sooty mangabeys (Cercocebus atys atys), J. Am. Assoc. Lab. Anim., 48, p. 192, 2009.

Wergård, E. M., Temrin, H., Forkman, B., Spångberg, M., Fredlund, H., and Westlund, K.: Training pair-housed Rhesus macaques (Macaca mulatta) using a combination of negative and positive reinforcement, Behav. Process., 113, 51-59, 2014.

Wergård, E. M., Westlund, K.., Spångberg, M., Fredlund, H., and Forkman, B.: Training success in group-housed long-tailed macaques (Macaca fascicularis) is better explained by personality than by social rank, Appl. Anim. Behav. Sci., submitted, 2015.
Westlund, K.: Can conditioned reinforcers and Variable-Ratio Schedules make food-and fluid control redundant? A comment on the NC3Rs Working Group's report, J. Neurosci. Meth., 204, 202-205, 2012a.

Westlund, K.: Questioning the necessity of food-and fluid regimes: Reply to Prescott and colleagues' response, J. Neurosci. Meth., 204, 210-213, 2012b.

Westlund, K.: Training is enrichment - and beyond, Appl. Anim. Behav. Sci., 152, 1-6, 2014.

Wolfensohn, S. and Honess, P.: Handbook of primate husbandry and welfare, Blackwell Publishing, Oxford, 2005.

Wolfle, T.: Laboratory animal technicians, Their role in stress reduction and human-companion animal bonding, The Veterinary clinics of North America, Small Animal Practice, 15, 449-454, 1985 . 